\title{
A minimal dark matter interpretation for the CRESST-II signal
}

\author{
Rainer Dick ${ }^{\mathrm{a}}$, Frederick S. Sage ${ }^{\mathrm{b}}$ \\ Department of Physics and Engineering Physics, University of Saskatchewan, 116 Science Place, Saskatoon, SK S7N 5E2, Canada
}

Received: 23 May 2012 / Published online: 25 July 2012

(C) The Author(s) 2012. This article is published with open access at Springerlink.com

\begin{abstract}
We report on the possible interpretation of the two proposed dark matter mass values $m_{\chi}=11.6 \mathrm{GeV}$ and $m_{\chi}=25.3 \mathrm{GeV}$ from CRESST-II within the framework of the Higgs portal minimal dark matter model. We find that the higher mass value yields a suitable fit with a dark matterHiggs coupling $\eta / 2=0.157$ and a recoil cross section which is compatible with contemporary estimates of the effective Higgs-nucleon coupling. On the other hand, the lower mass solution would require a large strangeness component in the nucleon to explain the corresponding nucleon recoil cross section reported by CRESST-II.
\end{abstract}

It is well known that DAMA and DAMA/LIBRA have reported for several years that they observe local dark matter through annual modulation of their detector signals [1, 2], whereas other direct search experiments have not found observable signal levels and reported exclusion limits instead. However, the announcements of the CoGeNT collaboration and of the CRESST-II collaboration that they may also have seen direct dark matter signals starts to tilt the balance, and it is intriguing that they all seem to indicate light dark matter in the mass range below $30 \mathrm{GeV}[3,4]$.

The CRESST-II collaboration reports that a maximum likelihood fit to its signal yields a dark matter mass of either $m_{\chi}=11.6 \mathrm{GeV}$ or $m_{\chi}=25.3 \mathrm{GeV}$ with a corresponding recoil cross section of $3.7 \times 10^{-5}$ pbarn or $1.6 \times 10^{-6}$ pbarn, respectively. We were intrigued by the question whether any of these mass values and corresponding recoil cross sections can be accommodated in the framework of a minimal dark matter model with Lagrange density [5-9],

$$
\begin{aligned}
\mathcal{L}= & -\frac{1}{2} \partial_{\mu} S \partial^{\mu} S-\frac{1}{2} m_{S}^{2} S^{2}-\frac{\lambda_{S}}{4} S^{4}-D_{\mu} H^{\dagger} D^{\mu} H \\
& -\frac{\eta}{2} S^{2}\left(H^{\dagger} H-\frac{v_{h}^{2}}{2}\right)-\frac{m_{h}^{2}}{2 v_{h}^{2}}\left(H^{\dagger} H-\frac{v_{h}^{2}}{2}\right)^{2} .
\end{aligned}
$$

\footnotetext{
a e-mail: rainer.dick@usask.ca

be-mail: fss325@mail.usask.ca
}

The scalar $S$ is a real electroweak singlet and $H$ is the Standard Model Higgs doublet. The electroweak singlet can therefore interact with Standard Model particles through Higgs exchange, hence the name Higgs portal for this type of models.

We would like to point out that the higher mass value $m_{\chi}=25.3 \mathrm{GeV}$ from CRESST-II is compatible with a minimal dark matter interpretation in the sense that the recoil cross section is compatible with standard effective Higgsnucleon couplings. On the other hand, the lower mass fit to the CRESST-II data could not be accommodated with (1) because even the highest contemporary estimates on the effective Higgs-nucleon coupling would imply too small a recoil cross section to explain the CRESST-II signal.

The parameter space of the models (1) is constrained both by perturbative stability [10-12] and by the theory of thermal dark matter creation (see e.g. [13-15] for the general theory of thermal creation of dark matter and $[6,11,16-19]$ for applications to minimal dark matter models), and if these constraints are combined with the nucleon recoil cross section limits from the null results of other direct dark matter search experiments like CDMS, EDELWEISS, PICASSO and XENON 100 [20-22], minimal dark matter with masses below $60 \mathrm{GeV}$ appears to be ruled out [23]. However, the high statistical significance reported by CRESST-II and the fact that it is by now the third experiment reporting a possible signal below $60 \mathrm{GeV}$ encouraged us to explore the consequences of assuming $m_{S}=m_{\chi}$ in (1). For the Higgs mass we used the range $m_{h}=124-126 \mathrm{GeV}$ from $[24,25]$. The variation of the Higgs mass over this small range did not change any numerical results up to two significant figures and most results not even to three significant figures. Therefore we report numerical results for $m_{h}=$ $125 \mathrm{GeV}$.

We found that (1) provided a satisfactory fit for the mass value $m_{S}=25.3 \mathrm{GeV}$ if $\eta=0.314$, in the sense that it could account for the observed dark matter density $\varrho_{D M}=$ $1170 \mathrm{eV} / \mathrm{cm}^{3}$, and that the resulting nucleon recoil cross section 
$\sigma_{S N}=\frac{g^{2} \eta^{2} v_{h}^{2}}{4 \pi m_{h}^{4}} \frac{m_{N}^{2}}{\left(m_{S}+m_{N}\right)^{2}}$

is compatible with the recoil cross section $\sigma_{S N}=1.6 \times$ $10^{-6}$ pbarn reported in [4].

The nucleon mass in (2) is chosen as $m_{N}=931.24 \mathrm{MeV}$, which is the average nucleon mass in long lived or stable tungsten isotopes. The required effective Higgs-nucleon coupling in (2) is then

$g v_{h}=318 \mathrm{MeV}$.

This coupling depends critically on the strangeness content

$y_{N}=\frac{2\langle N|\bar{s} s| N\rangle}{\langle N|\bar{u} u+\bar{d} d| N\rangle}$

of the nucleon. In their famous paper on the calculation of the effective Higgs-nucleon coupling, Shifman, Vainshtein and Zakharov had found $g v_{h}=210 \mathrm{MeV}$ under the assumption of negligible strangeness $y_{N} \ll 1$ in the nucleon [26]. In this case the complete effective Higgs-nucleon coupling arises from coupling of the Higgs to the three heavy quark species charm, bottom and top,

$\left.g v_{h}\right|_{y_{N} \ll 1}=\sum_{h=c, b, t} m_{h}\langle N|\bar{h} h| N\rangle=210 \mathrm{MeV}$,

where the heavy condensates arise from virtual fluctuations of the gluon sea in the nucleon.

The assumption $y_{N} \ll 1$ had been challenged by Cheng [27], who had argued for a large scalar strange form factor corresponding to $y_{N} \simeq 0.47$. Such a large strangeness contribution would increase the effective Higgs-nucleon coupling to $g v_{h}=530 \mathrm{MeV}$ because the relatively strong coupling $m_{s}\langle N|\bar{s} s| N\rangle / v_{h}$ of the Higgs to the strange quark overcompensates for the reduction of the heavy quark condensates which in turn arises from a reduction of the gluon content. There is wide consensus now that Cheng's original estimate of the strangeness content was too high and $y_{N}$ is likely between the extreme cases assumed in [26] and [27] and certainly smaller than 0.2 , see [28-31]. The value $g v_{h}=318 \mathrm{MeV}$ (3) corresponds to $y_{N}=0.16$.

On the other hand, the light mass solution $m_{S}=11.6 \mathrm{GeV}$ for the CRESST-II signal would require a Higgs-singlet coupling parameter $\eta=0.398$ to yield the correct dark matter density. Decreasing $m_{S}$ and increasing $\eta$ would seem to lower the required effective Higgs-nucleon coupling. However, this is overcompensated by the much larger recoil cross section $\sigma_{S N}=3.7 \times 10^{-5}$ pbarn which is required in the low mass fit to the CRESST-II signal, and the net effect would be a considerably larger effective Higgs-nucleon coupling $g v_{h}=577 \mathrm{MeV}$, which even exceeds Cheng's original estimate and requires a $y_{N}$ parameter that cannot be considered realistic.
In the terminology of [10] for mass parameters and coupling constants in a minimal real $\mathbb{Z}_{2}$ symmetric singlet model, $m_{S}=25.3 \mathrm{GeV}$ and $\eta=0.314$ corresponds to parameters $a_{2}=\eta / 2=0.157$ and $b_{2}=m_{S}^{2}-(\eta / 2) v_{h}^{2}=$ $-8.86 \times 10^{3} \mathrm{GeV}^{2}$ which seem to be compatible with perturbativity and vacuum stability at least up to the TeV scale if the singlet self-coupling is sufficiently large $\lambda_{S} \gtrsim 0.3$. However, minimal models will generically not be viable for extremely high energies. Extensions of minimal dark matter models (in a sense "modified minimal dark matter models" or "next to minimal dark matter models") have been proposed e.g. in [32-34], and supersymmetry is certainly also still an option.

In conclusion, the higher mass value $25.3 \mathrm{GeV}$ and the corresponding recoil cross section proposed by the CRESST-II collaboration can be accommodated in a minimal Higgs portal type dark matter model, but the alternatively proposed low mass value $11.6 \mathrm{GeV}$ certainly cannot be explained within such a model.

Acknowledgements This work was supported in part by NSERC Canada.

Open Access This article is distributed under the terms of the Creative Commons Attribution Noncommercial License which permits any noncommercial use, distribution, and reproduction in any medium, provided the original author(s) and source are credited.

\section{References}

1. R. Bernabei et al. (DAMA Collaboration), Eur. Phys. J. C 56, 333 (2008)

2. R. Bernabei et al. (DAMA Collaboration), Eur. Phys. J. C 67, 39 (2010)

3. C.E. Aalseth, J.I. Collar et al. (CoGeNT Collaboration), Phys. Rev. Lett. 107, 141301 (2011)

4. G. Angloher, F. Pröbst, J. Schmaler et al. (CRESST-II Collaboration), Eur. Phys. J. C 72, 1971 (2012)

5. V. Silveira, A. Zee, Phys. Lett. B 161, 136 (1985)

6. J. McDonald, Phys. Rev. D 50, 3637 (1994)

7. C.P. Burgess, M. Pospelov, T. ter Veldhuis, Nucl. Phys. B 619, 709 (2001)

8. H. Davoudiasl, R. Kitano, T. Li, H. Murayama, Phys. Lett. B 609, $117(2005)$

9. B. Patt, F. Wilczek, Higgs-field portal into hidden sectors, arXiv:hep-ph/0605188

10. M. Gonderinger, Y. Li, H. Patel, M.J. Ramsey-Musolf, J. High Energy Phys. 1001, 053 (2010)

11. S. Profumo, L. Ubaldi, C. Wainwright, Phys. Rev. D 82, 123514 (2010)

12. M. Gonderinger, H. Lim, M.J. Ramsey-Musolf, Complex scalar singlet dark matter: vacuum stability and phenomenology, arXiv:1202.1316v1 hep-ph

13. B.W. Lee, S. Weinberg, Phys. Rev. Lett. 39, 165 (1977)

14. P. Gondolo, G. Gelimini, Nucl. Phys. B 360, 145 (1991)

15. J. Edsjö, P. Gondolo, Phys. Rev. D 56, 1997 (1879)

16. R. Dick, R.B. Mann, K.E. Wunderle, Nucl. Phys. B 805, 207 (2008)

17. C.E. Yaguna, J. Cosmol. Astropart. Phys. 0903, 003 (2009) 
18. A. Goudelis, Y. Mambrini, C. Yaguna, J. Cosmol. Astropart. Phys. 0912, 008 (2009)

19. W.-L. Guo, Y.-L. Wu, J. High Energy Phys. 1010, 083 (2010)

20. Z. Ahmed, P. Di Stefano et al. (CDMS and EDELWEISS Collaborations), Phys. Rev. D 84, 011102 (2011)

21. S. Archambault, M.-C. Piro et al. (PICASSO Collaboration): Constraints on low-mass WIMP interactions with ${ }^{19} \mathrm{~F}$ from PICASSO, arXiv:1202.1240v1 [hep-ex]

22. E. Aprile, R.F. Lang, M. Schumann et al. (XENON100 Collaboration), Phys. Rev. Lett. 107, 131302 (2011)

23. A. Djouadi, O. Lebedev, Y. Mambrini, J. Quevillon, Phys. Lett. B 709, 65 (2012)

24. G. Aad et al. (ATLAS Collaboration), Phys. Rev. Lett. 108, 111803 (2012)

25. S. Chatrchyan et al. (CMS Collaboration), Phys. Lett. B 710, 26 (2012)
26. M.A. Shifman, A.I. Vainshtein, V.I. Zakharov, Phys. Lett. 78B, 443 (1978)

27. T.P. Cheng, Phys. Rev. D 38, 2869 (1988)

28. G.S. Bali, S. Collins et al. (QCDSF Collaboration), Phys. Rev. D 85, 054502 (2012)

29. R. Babich, R.C. Brower, M.A. Clark, G.T. Fleming, J.C. Osborn, C. Rebbi, D. Schaich, Phys. Rev. D 85, 054510 (2012)

30. S. Dinter, V. Drach, R. Frezzotti, G. Herdoiza, K. Jansen, G. Rossi, (ETM Collaboration): Sigma terms and strangeness content of the nucleon with $N_{f}=2+1+1$ twisted mass fermions, arXiv:1202.1480 [hep-lat]

31. A.W. Thomas, P.E. Shanahan, R.D. Young, Strangeness in the nucleon: what have we learned? arXiv:1202.6407 nucl-th

32. J.M. Cline, A.R. Frey, Phys. Rev. D 84, 075003 (2011)

33. J.M. Cline, A.R. Frey, Phys. Lett. B 706, 384 (2012)

34. M. Pospelov, A. Ritz, Phys. Rev. D 84, 113001 (2011) 